\title{
Safety and efficacy of asciminib treatment in chronic myeloid leukemia patients in real-life clinical practice
}

\author{
Valentín Garcia-Gutiérrez (1)', Alejandro Luna (D)', Juan M. Alonso-Dominguez ${ }^{2}$, Natalia Estrada ${ }^{3}$, Concepcion Boque ${ }^{4}$, \\ Blanca Xicoy ${ }^{3}$, Pilar Giraldo ${ }^{5}$, Anna Angona ${ }^{6}$, Alberto Alvarez-Larrán , Fermin Sanchez-Guijo ${ }^{8}$, María José Ramírez ${ }^{9}$, \\ Elvira Mora ${ }^{10}$, Patricia Vélez ${ }^{11}$, Ana Rosell ${ }^{12}$, Mercedes Colorado Araujo ${ }^{13}$, Beatriz Cuevas ${ }^{14}$, Miguel Sagüés ${ }^{3}$, \\ Montserrat Cortes ${ }^{15}$, Manuel Perez Encinas $\mathbb{E}^{16}$, Luis Felipe Casado Montero ${ }^{17}$, Melania Moreno Vega ${ }^{18}$, Luis Serrano ${ }^{19}$, \\ Valle Gomez ${ }^{20}$, Carmen Garcia-Hernandez ${ }^{21}$, Sunil Lakhwani2 ${ }^{22}$, Antonio Paz Coll ${ }^{23}$, Raquel de Paz ${ }^{24}$, \\ Sara Suarez-Varela ${ }^{25}$, Andrés Fernandez-Ruiz ${ }^{3}$, Raul Perez Lopez ${ }^{26}$, Almudena Ortiz-Fernández ${ }^{27}$, \\ Antonio Jiménez-Velasco ${ }^{28}$, Juan Luis Steegmann-Olmedillas ${ }^{20}$ and Juan Carlos Hernández-Boluda ${ }^{29}$
}

\section{Dear Editor,}

Despite the excellent overall survival (OS) of chronic myeloid leukemia (CML) patients, a significant proportion will fail currently available tyrosine-kinase inhibitors (TKIs) due to resistance or intolerance ${ }^{1-3}$. Intolerant patients are usually managed successfully with alternative second-generation tyrosine-kinase inhibitors (2GTKIs). However, more than half of the patients will eventually discontinue second-line treatment due to loss of response or toxicity ${ }^{4,5}$. Ponatinib is an effective drug in the setting of resistance to 2GTKIs, however with life-threatening side effects and varying responses ${ }^{6-8}$.

Asciminib is a first-in-class STAMP (Specifically Targeting the ABL Myristoyl Pocket) inhibitor that potently and specifically inhibits BCR-ABL1 via binding to a pocket distinct from the ATP binding site of the kinase . Asciminib has the potential to overcome resistance to prior TKIs, and also offers the possibility of dual inhibition of BCR-ABL1 in combination with ATP-binding $\mathrm{TKI}^{10,11}$. Asciminib has been evaluated in a phase I study in patients with Ph-positive leukemia failing prior TKIs, with promising results ${ }^{12}$.

\footnotetext{
Correspondence: Valentín Garcia-Gutiérrez (jvalentingg@gmail.com) ${ }^{1}$ Hematology, Hospital Universitario Ramón y Cajal. IRYCIS, Madrid, Spain ${ }^{2}$ Hospital Universitario Fundación Jiménez Díaz, Instituto de Investigación Sanitaria Fundación Jiménez Díaz (IIS-FJD), UAM, Madrid, Spain Full list of author information is available at the end of the article
}

Our aim is to share the first data on the use of asciminib in CML patients in clinical practice, allowed by Novartis under a managed-access program (MAP).

Data from $31 B C R-A B L 1$-positive CML patients treated with asciminib (median dose $40 \mathrm{mg}$ BID) between October 2018 and June 2020 in 25 institutions from the Spanish CML Group (GELMC) were retrospectively collected.

Monitoring and response milestones followed the European Leukemia Net 2020 recommendations ${ }^{13}$. All patients were treated under the MAP by Novartis. MAP requests were independently reviewed by a licensed treating physician to confirm that the following criteria were met: (a) treatment need of a serious or lifethreatening disease lacking commercially available options; (b) patient had to be ineligible or unable to participate in a clinical trial, and (c) the request should be in alignment with all applicable local laws and regulations. The study was approved by the Spanish Drug Agency and the Ethics Committee of the Hospital Universitario Ramón y Cajal (Madrid), with consent obtained from all the subjects. BCR-ABL1 analysis was not centralized but all samples were analyzed in EUTOS accredited laboratories.

Treatment emergent adverse events (TEAEs) were graded according to the National Cancer Institute Common Terminology Criteria for Adverse Events Version 4.0. Failure-free survival (FFS) is defined as time from first 
Table 1 Patients baseline characteristics.

\begin{tabular}{|c|c|}
\hline \multicolumn{2}{|l|}{ Patients' characteristics $(n=31)$} \\
\hline Age at data collection, yr (range) & $69(22-89)$ \\
\hline Age at diagnosis, yr & 55 \\
\hline Female sex \% (n) & $65(20)$ \\
\hline Time on previous TKIs, months (range) & $77(13-221)$ \\
\hline \multicolumn{2}{|l|}{ Disease stage before asciminib \% (n) } \\
\hline Chronic phase & $96(30)$ \\
\hline Accelerated phase & $3(1)$ \\
\hline Blast phase & 0 \\
\hline \multicolumn{2}{|l|}{ Sokal risk \% (n) } \\
\hline Low & $32(10)$ \\
\hline Intermediate & $36(11)$ \\
\hline High & $33(10)$ \\
\hline \multicolumn{2}{|l|}{ TKI at diagnosis \% (n) } \\
\hline Imatinib & $74(23)$ \\
\hline Dasatinib & $10(3)$ \\
\hline Nilotinib & $13(4)$ \\
\hline Bosutinib & $3(1)$ \\
\hline Ponatinib & $0(0)$ \\
\hline$\geq 3$ prior TKI lines $\%(n)$ & $90(28)$ \\
\hline Prior use of ponatinib \% ( $n)$ & $35(11)$ \\
\hline BCR-ABL1 mutations \% ( $n)$ & $38(12)$ \\
\hline E255K \% (n) & $25(3)$ \\
\hline Exon $7 \%(n)$ & $17(2)$ \\
\hline T315। \% (n) & $8(1)$ \\
\hline Others \% (n) & $50(6)$ \\
\hline
\end{tabular}

dose of asciminib to on-treatment death, progression to advanced phase, confirmed loss of complete cytogenetic response (CCyR), loss of complete hematologic response (CHR), treatment discontinuation for any reason (intolerance or lack of efficacy), or death for any reason. Progression-free survival (PFS) is defined as time from first dose of asciminib to on-treatment death, progression to advanced phase, loss of CHR, or death for any reason. Variables studied to identify factors associated with response to asciminib included resistance versus intolerance, resistance to previous TKIs, previous use of ponatinib, prior $\mathrm{CCyR}$ status, and presence of $B C R-A B L 1$ mutations.

Median time on previous TKI treatment until asciminib initiation was 77 months (range 6-221 months). Patients' baseline characteristics are compiled in Table 1. Patients were heavily pretreated with 28 of them having received 3 or more TKIs previous to asciminib. Eleven patients (35\%) had been treated with ponatinib. Twelve patients (39\%) had baseline BCR-ABL1 mutations (1 case with T315I mutation). Switch to asciminib was due to intolerance to prior TKIs in 22 patients and due to resistance in the remaining 9. Starting asciminib dose was $40 \mathrm{mg}$ BID in all patients except in the single case harboring the T315I mutation, who received $200 \mathrm{mg}$ BID.

Median duration on asciminib treatment at time of analysis was 8.8 months (8.2 and 9.3 in resistant and intolerant patients, respectively). Median follow-up for the entire population was 10.2 months.

Overall, $58 \%$ of patients had $\geq 1$ TEAE of any grade and $32 \%$ had $\geq 1$ TEA of grade $3-4$. Thirteen patients (42\%) experienced mild non-hematological side effects (grade 12 ), the most frequent being fatigue (19\%), joint pain (16\%), and nausea (9\%). Four patients $(12 \%)$ had severe (grade $3-$ 4) non-hematological events: fatigue, hepatotoxicity, hypertension, and pericardial effusion (1 patient each). Three patients $(9.7 \%)$ developed grade 4 thrombocytopenia (all of them had previously experienced same toxicity with previous TKIs), 2 of them combined with grade 4 neutropenia. One of the 4 patients who had suffered from pancreatitis on prior TKI treatment presented the same AE under asciminib, which resolved following treatment discontinuation. The patient receiving $200 \mathrm{mg}$ BID due to T315I mutation suffered a total of three grade 1 AEs: thrombocytopenia, fatigue, and abdominal pain.

Dose reduction (median reduced dose was $40 \mathrm{mg}$ once a day), mostly due to hematological toxicity, was required in 9 patients (29\%), with temporary treatment interruption in 7 cases. Probabilities of suffering grade 3-4 TEAE were $44 \%$ and $22 \%$ for resistant and intolerant patients, respectively.

Four patients discontinued treatment due to progression to blast phase $(n=2)$ and lack of efficacy $(n=2)$. No patients discontinued treatment due to TEAEs. 2 patients died during the observation period due to progression. At last evaluation, 27 patients $(87 \%)$ remained on asciminib treatment.

At the time of analysis, with a median time on asciminib of 8.8 months, the cumulative responses rates (including reaching or at least maintaining previous response) of $\mathrm{CHR}, \mathrm{CCyR}$ and major molecular response (MMR) were $100 \%, 66 \%$, and $41 \%$, respectively. A total of 17 patients (55\%) and 28 patients (90\%) improved or maintained baseline response, respectively during follow-up (Table 2).

In patients without the respective responses at baseline, cumulative rates of CCyR and MMR were 48\% (8/17) and $33 \%$ (8/24), respectively. Probabilities to obtain CCyR and MMR in resistant and intolerant patients were 33\% (3/9) vs $62 \%(5 / 8)$ and $11 \%(1 / 9)$ vs $47 \%$ (7/15), respectively.

Amid the 11 patients previously treated with ponatinib, 3 patients (27\%) showed improvement of response 
Table 2 Response to asciminib subanalysis regarding baseline response.

\begin{tabular}{cccc}
\hline & Resistant (9) & Intolerant (19*) & Total (29*) \\
\hline Best response to asciminib & & \\
All patients & & \\
CHR $^{\mathrm{a}}$ & $9 / 9(100 \%)$ & $19 / 19(100 \%)$ & $29 / 29(100 \%)$ \\
CCyR $^{\mathrm{a}}$ & $3 / 9(33 \%)$ & $16 / 19(84 \%)$ & $19 / 29(66 \%)$ \\
MMR $^{\mathrm{a}}$ & $1 / 9(11 \%)$ & $11 / 19(58 \%)$ & $12 / 29(41 \%)$ \\
MR4.5 $^{\text {a }}$ & $0 / 9(0 \%)$ & $4 / 19(21 \%)$ & $4 / 29(14 \%)$ \\
Patients without response at baseline & \\
CCyR $^{\mathrm{b}}$ & $3 / 9(33 \%)$ & $5 / 8(63 \%)$ & $8 / 17(47 \%)$ \\
MMR $^{\mathrm{b}}$ & $1 / 9(11 \%)$ & $7 / 15(47 \%)$ & $8 / 24(33 \%)$ \\
MR4.5 $^{\mathrm{b}}$ & $0 / 3(0 \%)$ & $4 / 19(21 \%)$ & $4 / 29(14 \%)$ \\
\hline
\end{tabular}

*Due to short follow-up, 2 patients were excluded from response analysis. CHR complete hematological response, CCyR complete cytogenetic response, $M M R$ major molecular response, MR4.5 detectable disease with BCR-ABL1IS < $0.0032 \%$.

a Patients with CHR, CCyR, MMR, or MR4.5 at baseline were evaluable for hematologic, cytogenetic, or molecular response and were considered responders if they maintained their response. ${ }^{b}$ Evaluable patients without a CCyR, MMR, or MR4.5 at baseline.

achieving at least MMR. Two were in TKI-intolerant group and one in the TKI-resistant group.

Regarding the 12 patients with $B C R-A B L 1$ baseline mutations, 4 patients (44\%) improved baseline response: 2 patients (carrying M344I and E255V mutations reached CCyR and 2 patients improved their responses to MMR (both carrying exon 7 deletion). The patient with T315I mutation suffered eventually from CHR loss after 3 months and asciminib was interrupted due to progression. With a median follow-up of 10.2 months, the estimated FFS, PFS, and OS were $87 \%, 94 \%$, and $94 \%$, respectively.

We analyzed the factors associated with the response to asciminib, identifying the achievement of $\mathrm{CCyR}$ with prior TKI treatment as the only predictive factor (OR 10.5; 95\% CI: 1.02-108.6).

To our knowledge, the present study is the first to describe the results of asciminib treatment in CML patients managed in clinical practice under a compassionate access program. It is important to highlight the differences between our study population and that of the previously published paper from the phase I clinical trials with asciminib $^{12}$. In the phase I trial, patients could receive asciminib after failing at least two TKIs, while in the present study patients did not have any alternative effective treatment. Taking into consideration that the majority of patients in our study were classified as intolerant to previous TKIs, the population should be considered as at special high risk of suffering side effects. Our data confirmed the good safety profile of asciminib in a heavily treated real-world population. No patients discontinued asciminib due to side effects, which is in line with previous asciminib studies (with shorter follow-up) that showed probabilities of treatment discontinuations due to side effects to be less than $10 \%{ }^{11}$. This information contrast with data from 2GTKI studies in which probabilities to discontinue treatment due to side effects are higher ${ }^{6}$. Besides good data regarding asciminib tolerability, our study suggests how patients that suffer from pancreatitis or hematological toxicities with previous TKIs are at risk of suffering same side effects with asciminib.

Regarding efficacy, overall responses to asciminib were similar to the ones in phase 1 trial, with $61 \%$ of patients achieving or maintaining CCyR. Despite adequate overall responses, it is important to mention that probabilities to achieve CCyR in resistant patients without $\mathrm{CCyR}$ at baseline was 33\% (3/9), which is significantly lower compared to results observed in phase I trial. One explanation could be the more pretreated population in our study; however, ongoing studies will clarify probabilities of achieving optimal responses in patients without baseline responses.

Our study included $30 \%$ of patients that had received ponatinib prior to asciminib. As expected, only 3/9 patients show improved responses, with 2 of these patients corresponding to the intolerance group. The phase I study also included $30 \%$ of patients previously treated with ponatinib, nevertheless, treatment responses according to previous ponatinib exposure were not reported to date. We believe that further asciminib studies should describe asciminib activity in ponatinib-resistant patients in order to clarify best treatment alternatives in 2GTKI-resistant patients ${ }^{14}$.

In conclusion, the results presented, in line with data from the phase 1 study, show asciminib as a safe and efficacious drug for CML patients without treatment alternatives in common clinical practice. Further ongoing studies will provide information related the type of patients that would benefit the most from this new treatment approach.

\footnotetext{
Author details

${ }^{1}$ Hematology, Hospital Universitario Ramón y Cajal. IRYCIS, Madrid, Spain. ${ }^{2}$ Hospital Universitario Fundación Jiménez Díaz, Instituto de Investigación Sanitaria Fundación Jiménez Díaz (IIS-FJD), UAM, Madrid, Spain. ${ }^{3}$ Institut Catala d'Oncologia, Hospital Germans Trias i Pujol, Josep Carreras Leukemia Research Institute, Universitat, Autònoma de Barcelona, Badalona, Spain. ${ }^{4}$ Institut Catala d'Oncologia - L'Hospitalet de Llobregat, L'Hospitalet de Llobregat, Spain. ${ }^{5}$ Hospital Quiron Zaragoza, Zaragoza, Spain. ${ }^{6}$ Hematology, Institut Catala d'Oncologia, Girona, Spain. ${ }^{7}$ Hematology Department, Hospital Clínic, Barcelona, Spain. ${ }^{8}$ Hematology Department, IBSAL-Hospital Universitario de Salamanca, CIC and CIBERONC, University of Salamanca, Salamanca, Spain. ${ }^{9}$ Hematology Department, Hospital de Jerez de la Frontera, Jerez, Spain. ${ }^{10}$ Hematology Department, Hospital Universitario y Politécnico La Fe, Valencia, Spain. ${ }^{11}$ Hospital Mutua Terrassa, Terrassa, Spain. ${ }^{12}$ Hematology, Hospital Virgen de la Victoria, Malaga, Spain. ${ }^{13}$ Hospital U. Marqués de Valdecilla, Servicio de Hematología-Hemoterapia, Santander, Spain. ${ }^{14}$ Hematology,
} 
Hospital Universitario de Burgos, Burgos, Spain. ${ }^{15} \mathrm{Hospital}$ General de Granollers, Barcelona, Spain. ${ }^{16}$ Hospital Clínico Universitario de Santiago de Compostela, Santiago de Compostela, Spain. ${ }^{17}$ Hospital Virgen de la Salud, Toledo, Madrid, Spain. ${ }^{18}$ Hospital Doctor José Molina Orosa de Lanzarote, Arrecife, Spain. ${ }^{19}$ Hospital General de Castellón, Castellón, Spain. ${ }^{20}$ Hospital Universitario La Princesa, Madrid, Spain. ${ }^{21}$ Hospital General de Alicante, Alicante, Spain. ${ }^{22}$ Department of Hematology, Hospital Universitario de Canarias, La Laguna, La Laguna, Spain. ${ }^{23}$ Hospital Universitario Puerto Real, Puerto Real, Spain. ${ }^{24}$ Hematology Department, Hospital Universitario La PazIdipaz, Madrid, Spain. ${ }^{25}$ Hospital de Jerez de la Frontera, Jerez de la Frontera, Spain. ${ }^{26}$ Servicio de Hematología, Hospital Universitario Clínico Virgen de la Arrixaca, Murcia, Spain. ${ }^{27}$ Instituto Ramón y Cajal de Investigación Sanitaria (IRYCIS), Hospital Universitario Ramón y Cajal, Madrid, Spain. ${ }^{28}$ Hospital Regional Universitario de Málaga, Málaga, Spain. ${ }^{29}$ Hospital Clinico de Valencia, Valencia, Spain

\section{Author contributions}

V.G.G., A.L., and J.L.S. designed the study, coordinated the group, and wrote the manuscript. J.H.B. contributed to clinical data collection and wrote the manuscript. J.M.A.D. contributed with statistical analysis. The other authors contributed to clinical data collection and reviewed the manuscript.

\section{Conflict of interest}

V.G.G.: Novartis, Incyte, Pfizer and BMS - Speaker Honoraria, advisory committees and research funding; J.M.A.D.: Research funding from Incyte, Celgene, Pfizer, Astellas. BMS - Speaker Honoraria, advisory committees; Pfizer Speaker Honoraria, advisory committees. Incyte - Advisory committees; J.L.S.: Novartis - Consultancy, Speaker Honoraria, advisory committees; research grants; BMS - Consultancy, Speaker Honoraria, advisory committees; research grants; Pfizer - Consultancy, Speaker Honoraria, advisory committees; research grants: Incyte - Advisory committees. S.L.L.: Novartis - Speaker honoraria, advisory committees; BMS - Speaker honoraria. The rest of the authors declare that they have no conflict of interest.

\section{Publisher's note}

Springer Nature remains neutral with regard to jurisdictional claims in published maps and institutional affiliations.

Received: 9 November 2020 Revised: 13 December 2020 Accepted: 15 January 2021

Published online: 09 February 2021

\section{References}

1. García-Gutiérrez, V. \& Hernández-Boluda, J. C. Current treatment options for chronic myeloid leukemia patients failing second-generation tyrosine kinase inhibitors. J. Clin. Med. 9, 2251 (2020).

2. Ibrahim, A. et al. Efficacy of tyrosine kinase inhibitors (TKIs) as third-line therapy in patients with chronic myeloid leukemia in chronic phase who have failed 2 prior lines of TKI therapy. Blood 116, 5497-5500 (2010).

3. Hochhaus, A. et al. Long-term outcomes of imatinib treatment for chronic myeloid leukemia. N. Engl. J. Med. 376, 917-927 (2017).

4. García-Gutiérrez, V. \& Hernández-Boluda, J. C. Tyrosine kinase inhibitors available for chronic myeloid leukemia: efficacy and safety. Front Oncol. 9, 603 (2019).

5. Hochhaus, A. et al. Bosutinib for pretreated patients with chronic phase chronic myeloid leukemia: primary results of the phase 4 BYOND study. Leukemia 34, 2125-2137 (2020).

6. Cortes, J. et al. Ponatinib efficacy and safety in Philadelphia chromosomepositive leukemia: final 5-year results of the phase 2 PACE trial. Blood 132 , 393-404 (2018)

7. Hochhaus, A. et al. Expert opinion-management of chronic myeloid leukemia after resistance to second-generation tyrosine kinase inhibitors. Leukemia 34, 1495-1502 (2020).

8. Breccia, M. et al. Identification, prevention and management of cardiovascular risk in chronic myeloid leukaemia patients candidate to ponatinib: an expert opinion. Ann. Hematol. 96, 549-558 (2017).

9. Manley, P. W., Barys, L. \& Cowan-Jacob, S. W. The specificity of asciminib, a potential treatment for chronic myeloid leukemia, as a myristate-pocket binding $A B L$ inhibitor and analysis of its interactions with mutant forms of BCR-ABL1 kinase. Leuk. Res. 98, 106458 (2020).

10. Wylie, A. A. et al. The allosteric inhibitor ABL001 enables dual targeting of BCRABL1. Nature 543, 733-737 (2017).

11. Eadie, L. N., Saunders, V. A., Branford, S., White, D. L. \& Hughes, T. P. The new allosteric inhibitor asciminib is susceptible to resistance mediated by ABCB1 and ABCG2 overexpression in vitro. Oncotarget 9, 13423-13437 (2018).

12. Hughes, T. P. et al. Asciminib in chronic myeloid leukemia after ABL kinase inhibitor failure. N. Engl. J. Med. 381, 2315-2326 (2019).

13. Hochhaus, A. et al. European LeukemiaNet 2020 recommendations for treating chronic myeloid leukemia. Leukemia 34, 966-984 (2020).

14. Novartis. Novartis investigational novel STAMP inhibitor asciminib (ABL001) meets primary endpoint of Phase III chronic myeloid leukemia study [Press release, 26 August 2020]. Available at https://www.novartis.com/news/mediareleases/novartis-investigational-novel-stamp-inhibitor-asciminib-abl001meets-primary-endpoint-phase-iii-chronic-myeloid-leukemia-study (Accessed: 30 September 2020). 